\title{
Fusion d'un cours de chimie et de physique par l'apprentissage par problèmes (APP) : mise en place, améliorations et incidences chez les étudiants
}

\author{
Celine DARIE \\ Polytech Grenoble, Université Grenoble Alpes, celine.darie@univ-grenoble-alpes.fr \\ CHRISTOPHE DURAND \\ Polytech Grenoble, Université Grenoble Alpes, christophe.durand@univ-grenoble-alpes.fr
}

\section{Résumé}

La démarche de fusion de deux cours théoriques a pour objectif de donner plus de sens réciproquement à chacun d'eux pour une meilleure adhésion et une meilleure réussite aux examens des étudiants. La mise en œuvre d'une méthodologie APP (Apprentissage par problèmes) a été retenue comme préalable. Une expérimentation sur trois ans du dispositif est décrite avec ses évolutions et l'analyse des résultats notamment sur ces deux points : l'adhésion des étudiants et la réussite aux examens.

\begin{abstract}
The objective of merging two theoretical courses is to give each of them more meaning for better adherence and better success in student exams. The implementation of an APP (Problembased Learning) methodology was retained as a prerequisite. A three-year experiment of the device is described with its developments and the analysis of the results, in particular on these two points: student adherence and exam success.
\end{abstract}

\section{Mots-clés}

Fusion de deux cours, liaisons chimiques, physique du solide, apprentissage par problèmes

\section{Keywords}

Merging of two theoretical courses, chemical bonds, solid-state physics, problem-based learning 


\section{Introduction}

Initié dans les années 1970 pour des formations médicales, les pratiques pédagogiques basées sur la méthode APP (Apprentissage Par Problèmes) se sont progressivement développées dans l'enseignement supérieur. Aujourd'hui, cette méthode pédagogique du type socioconstructiviste est employée dans de nombreux pays, comme la Belgique ou le Canada (Bédard et Raucent, 2015 ; Raucent et Milgrom, 2013) et pour des matières très diverses. En particulier, l'APP a été mis en place notamment dans des écoles d'ingénieurs (Raucent, Braibant, Theux, Jacqmot, Milgrom, Vander, Borght et Wouters, 2004 ; Boutin, Dalle, Lachiver et Mabilleau, 2008) et a montré des effets bénéfiques sur la motivation et la méthodologie de travail des étudiants (Galand, Bourgeois, Frenay et 2005 ; Bédard, Lison, Dalle et Boutin, 2010). En France, ce type de pratique tend à se développer de plus en plus depuis une dizaine d'années. Des exemples d'APP en physique ont aussi été réalisés avec succès pour développer l'esprit critique et l'analyse des données (Celik, Onder et Silay, 2011 ; Mundilarto, 2017).

L'expérimentation présentée décrit la mise en œuvre d'une approche APP au sein de l'école d'ingénieur Polytech Grenoble dans la filière «Matériaux » qui fusionne deux cours théoriques: l'un en chimie intitulé «Liaisons Chimiques » et l'autre en physique nommé « Physique du Solide ». Ces deux cours réalisés de manière traditionnelle sous la forme de cours magistraux et de TD étaient bien appréciés des étudiants selon les évaluations des enseignements (appréciation globale supérieure à 4/5). Cependant, la compréhension de ces deux cours était pour une majorité d'étudiants très partielle avec une moyenne aux examens de $11,4 / 20$ et plus de $30 \%$ des étudiants ayant une note inférieure à 10/20. Ces résultats décevants aux examens nous ont motivés à adopter une nouvelle approche pédagogique avec notamment la mise en place d'un enseignement en APP fusionnant les cours de chimie et de physique qui expliquent respectivement la formation des molécules et des solides à partir de la physique quantique. À terme, ces changements ont pour objectif d'améliorer la compréhension de ces deux cours théoriques.

L'implantation du dispositif décrite ici correspond à une expérimentation itérative menée au cours de trois années consécutives. Dans une première partie seront présentés le contexte et la problématique avec une description des objectifs. Dans la partie suivante, le dispositif mis en place la première année et les évolutions réalisées les années suivantes, ainsi que la méthodologie de l'évaluation du dispositif sont décrits. Enfin, dans une troisième partie, les 
résultats des analyses et un bilan sur les apprentissages sur les trois années d'expérimentations seront présentés et discutés.

\section{Contexte et problématique}

Avant 2015, les enseignements de "Liaisons chimiques» et de "Physique du Solide» correspondaient à deux enseignements distincts confiés à des enseignants de Chimie et de Physique en première année de la filière «Matériaux» de l'école d'Ingénieur Polytech Grenoble (équivalent à une $3^{\mathrm{e}}$ année de Licence) pour un effectif d'une cinquantaine d'étudiants par an. Pourtant, ces deux cours se basent sur l'application de la physique quantique pour décrire le comportement des électrons et utilisent des concepts et des méthodes de résolution parfois similaires, mais sous un angle et un vocabulaire différents. Des notions totalement nouvelles et très abstraites sont introduites ce qui est source d'importants blocages dans ces deux enseignements. Il s'ensuit parfois un désintéressement de certains étudiants pour « ces »matières jugées trop théoriques dans le cadre d'une formation d'ingénieur. Pourtant, elles sont absolument essentielles pour une formation d'ingénieurs en «Matériaux », puisqu'elles expliquent notamment la formation des molécules et des solides, ainsi que leurs propriétés physiques. Pour améliorer la compréhension et l'adhésion des étudiants à ces deux matières théoriques, nous avons entrepris, pendant l'année universitaire 2016/2017, les deux changements suivants :

1. la fusion de ces deux cours qui permet d'introduire la continuité des concepts et des méthodes en passant de la construction d'une molécule à quelques atomes jusqu'à la formation des solides réunissant un très grand nombre d'atomes ;

2. la mise en place d'une pédagogie active sous la forme d'un APP pour renforcer la compréhension et faire manipuler aux étudiants des concepts théoriques nouveaux.

Nous détaillons ci-après les raisons de ces deux choix dans la mise en place du nouveau dispositif pédagogique.

\subsection{Choix de fusion d'un cours de chimie et de physique}

Bien que différents, le cours de chimie sur les liaisons chimiques et celui de physique sur la physique du solide butent sur le même obstacle épistémologique (Bachelard, 1938), celui remettant en cause le modèle de l'atome isolé, qui est profondément ancré chez les étudiants. En effet, ces 2 cours apportent une nouvelle vision de l'atome qui n'est plus celle d'un atome 
isolé, mais en interaction avec les autres atomes, ce qui impose de changer totalement sa description.

Pour lever les résistances à ce nouveau modèle de l'atome non isolé, nous avons fait le choix de partir du système le plus simple à 2 atomes (molécule d'hydrogène $\mathrm{H}_{2}$ ) et d'augmenter progressivement le nombre d'atomes en interaction : 4 atomes de carbone (molécule de butadiène), 22 atomes de carbone (molécule de carotène), 40 millions d'atomes (chaîne polymère de polybutadiène) et enfin des milliards de milliards d'atomes (solide de cuivre ou d'or). Cette démarche qui propose une gradation en continuité du modèle de l'atome non isolé a été rendue possible grâce à la fusion de ces deux cours. Cette nouvelle approche épistémologique a l'avantage d'améliorer la compréhension intrinsèque de ces deux matières et permet en même temps de faire du lien entre elles, puisque les mêmes équations sont résolues à des échelles différentes. Il faut toutefois veiller à utiliser des termes et des notations similaires pour assurer cette continuité. Cette continuité épistémologique par la fusion de ces deux disciplines est originale et oblige à jongler entre des ouvrages de référence de chimie du solide (McQuarrie et Simon, 2000) et physique du solide (Kittel, 2007).

Une autre difficulté rencontrée par les étudiants est le passage aux très grands nombres dans le cas des solides, car cela nécessite d'introduire un nouveau formalisme pour pouvoir décrire le comportement de milliards d'atomes en interaction. Pour donner une image, nous passons de la description de quelques gouttes d'eau à celle de la mer où l'eau se déplace sous forme de vagues. L'approche graduelle en partant du «petit» pour aller vers «l'infiniment grand» permet de montrer que le formalisme établi pour quelques atomes devient inutilisable face aux très grands nombres, et in fine de comprendre la nécessité d'introduire de nouveaux concepts pour opérer ce changement d'échelle. Cela dit, il importait également de choisir le bon levier pédagogique pour nous permettre de présenter cette nouvelle conception de l'atome. Ce choix est présenté dans la prochaine section.

\subsection{L'Apprentissage par problèmes (APP)}

Le choix de la méthode APP a été instrumental pour ce travail (Bédard, Bibeau, Pilon et Turgeon, 2020). Ce choix de pédagogie active du type socio-constructiviste a pour but de dépasser l'obstacle épistémologique du modèle de l'atome non isolé en marquant les esprits des étudiants à travers des conflits cognitifs pour qu'ils changent en profondeur leurs représentations et s'approprient les nouveaux concepts. Les étudiants sont principalement en position d'apprentissage, puisque le cours n'est plus « dispensé » par l'enseignant, mais est mis 
à disposition pour être travaillé dans l'objectif de résoudre des situations-problèmes. Une attention particulière porte sur la construction des situations-problèmes (Boilevin, 2005) qui doivent être motivantes pour les étudiants et qui ciblent précisément les nouvelles notions à apprendre. La coopération active au sein du groupe est un des éléments clefs de la réussite de cette méthode pour pouvoir justement surmonter les conflits cognitifs induits par cette méthode socio-constructiviste. Ainsi pour comprendre et mettre du sens sur les nouvelles notions qui sont souvent difficiles à intégrer, la coopération au sein du groupe joue un rôle indispensable : il est notamment intéressant de voir la construction d'une solution entraîner peu à peu les étudiants à mettre en doute leurs certitudes et à proposer d'autres approches conceptuelles (voulues par le problème). «La synthèse permet de mettre en commun les explications émises par chaque étudiant. Elle se base sur les informations récoltées, les expériences réalisées... Il s'ensuit un débat d'idées, où, pour répondre au problème posé, chaque étudiant doit argumenter son approche de solution, justifier sa position, convaincre... ou se laisser convaincre » (Aguirre, Jacqmot, Milgrom, Raucent, Soucisse, Vander et Borght, 2000).

\subsection{Les objectifs du nouveau dispositif pédagogique}

Dans ce contexte, la fusion de ces deux matières en intégrant une méthode d'apprentissage actif avait deux objectifs :

- Obtenir une meilleure adhésion des étudiants pour ces deux matières théoriques qui sont d'une grande importance dans la formation d'ingénieur en matériaux ;

- Favoriser la compréhension du modèle de l'atome non isolé et, conséquemment, améliorer le taux de réussite aux examens pour ces deux matières.

Ce dispositif pédagogique basé sur quatre séquences APP a été joué une première fois pendant l'année universitaire 2016-2017 et a ensuite été amélioré les deux années suivantes (2017-2018 et 2018-2019). Pour répondre au $1^{\mathrm{er}}$ objectif, nous avons recueilli le ressenti des étudiants sous la forme d'un questionnaire détaillé anonyme à la fin de chaque année. Il permettait notamment de connaître leur adhésion au contenu enseigné et au dispositif APP. Afin de répondre au $2^{\mathrm{e}}$ objectif, nous avons collecté les notes des examens terminaux dont le contenu et la forme sont restés inchangés pour les comparer aux notes obtenues avec l'approche classique cours/TD. Pour connaître l'impact du dispositif pédagogique, nous étudierons les réponses aux questionnaires et nous comparerons les notes avant et après la mise en place du dispositif pédagogique. 


\section{Caractéristiques du dispositif APP et de son évaluation}

Le dispositif pédagogique APP a été mis en place pour une classe d'environ 50 étudiants répartis en 8 groupes de 6-7 étudiants. Pour rappel, le principe de la méthode APP repose sur le principe brièvement décrit ci-après (Raucent et Milgrom, 2013). Les étudiants, regroupés par équipes, travaillent ensemble à résoudre un problème pour lequel ils n'ont reçu aucune formation au préalable. La découverte du problème se fait collectivement lors de la séance « Aller ». Ce travail de groupe permet de bien comprendre le problème posé et de mettre en commun les préconceptions des étudiants sur le problème donné. Dans un second temps, chaque étudiant travaille seul de manière autonome à essayer de résoudre le problème par lui-même (Séance de TRAvail Personnel que nous appelons « TRAP »). Pendant ce temps, il s'appuie sur des documents de cours transmis par les enseignants. Dans un troisième temps, lors d'une séance en groupe (Séance « Retour»), les solutions de chaque étudiant sont discutées afin que le groupe puisse proposer une seule solution qui soit construite collectivement et qui fasse consensus. Pour faciliter ce travail en équipe, une organisation est proposée avec différents rôles (animateur, scribe, maître du temps et secrétaire) pour fluidifier le partage des idées, de la parole et être efficace pour produire la solution. La démarche est accompagnée par l'enseignant qui joue un rôle de tuteur. La résolution d'un problème a pour objectif de susciter une motivation pour apprendre le contenu du cours et favoriser les apprentissages en profondeur de notions difficiles (Svinicki et McKeachie, 2014). À la fin, il y a un mini-examen individuel (que nous appellerons quick-test) pour s'assurer que chaque membre du groupe a acquis les apprentissages visés. Nous allons détailler, dans la suite, les spécificités de l'approche APP mise en place et le procédé d'évaluation de cet enseignement par les étudiants.

\subsection{Caractéristiques du dispositif lors de la première année d'expérimentation (2016/2017)}

Une organisation sur 6 semaines a été choisie durant lesquelles les étudiants doivent résoudre quatre séquences APP avec des situations-problèmes différentes. Comme illustré sur la Figure 1, le plan de séquençage du dispositif est le suivant :

- APP 0 : «Apporter votre grain de sable!»;

- APP 1 : «Les deux font la paire »;

- APP 2 : «Le carbone dans tous ses états»;

- APP 3 : «À plusieurs, on est plus fort»;

- APP 4 : « Mind the gap! ». 
Au préalable, il y a une initiation au travail de groupe et à la méthodologie APP (séance appelée APP0) qui sera décrite ci-après. Les deux premiers APP (APP1 et APP2) sont liés au cours de Liaisons chimiques et les deux derniers (APP3 et APP4) correspondent au cours de Physique du Solide.

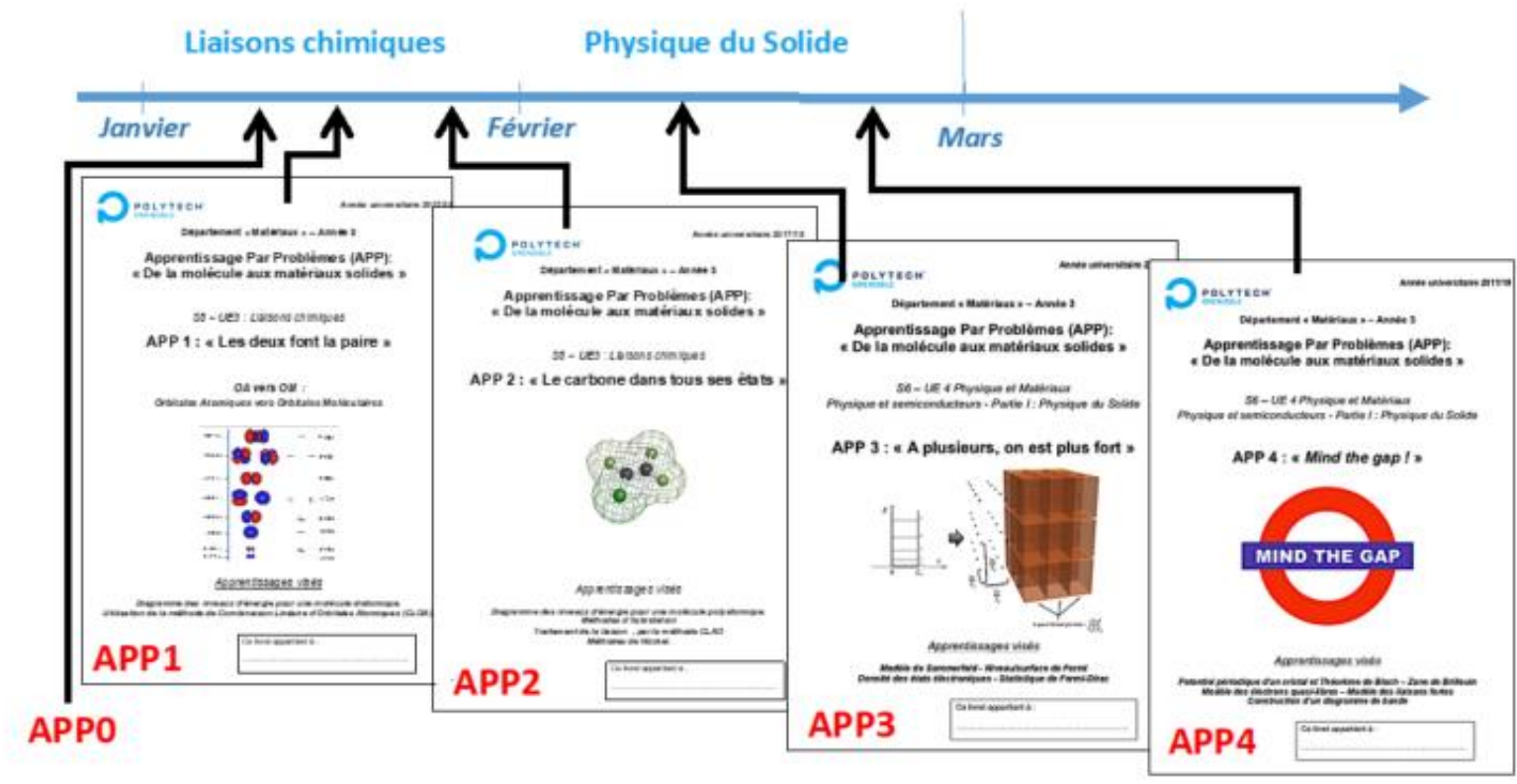

Figure 1 : Déroulement du dispositif APP « De la molécule aux matériaux solides » qui fusionne deux enseignements différents : «Liaisons Chimiques » correspondant aux APP 1 et 2 et «Physique du Solide » correspondant aux APP 3 et 4

Pour la première expérimentation, un certain nombre de choix pédagogiques et organisationnels ont été faits pour rendre le dispositif APP le plus efficace possible. Ils sont décrits ci-dessous :

- La mise en place d'une activité sur $2 \mathrm{~h}$ (APP0) pour apprendre à travailler en groupe et pour introduire la méthodologie APP. Pour les besoins de la matière, un sujet autour des grands nombres a été choisi. Le défi proposé aux étudiants propose de «calculer le nombre de grains de sable qu'il y a sur la Terre ». Ce sujet a l'avantage d'être très simple à comprendre et semble au premier abord très compliqué à résoudre. Afin de bien illustrer que travailler en groupe n'est pas inné, l'activité est divisée en deux défis similaires avec des modalités différentes. Le premier défi est fait sans aucune consigne. Le second défi est par contre bien organisé en imposant une méthodologie APP (travail personnel préalable, travail sur des ressources, attribution de rôle, etc.). Une comparaison du vécu des deux mises en situation permet aux étudiants de comprendre l'importance de la méthode et de l'organisation des séances pour rendre le travail de 
groupe efficace et pour favoriser l'apprentissage individuel de tous les membres du groupe ;

- Le temps du travail personnel (TRAP) n'est pas à faire à la maison, mais il est directement inclus dans les séances. Ainsi, les enseignants-tuteurs peuvent s'assurer que le travail personnel est bien fait de manière individuelle. Par ailleurs, les enseignantstuteurs sont présents pour répondre à titre individuel aux questions des étudiants ou aider les étudiants en grande difficulté qui restent complètement bloqués, afin qu'ils ne perdent pas ce temps à ne rien faire. L'intégration du travail personnel avec présence des enseignants-tuteurs permet de lutter contre l'écueil récurrent des APP, où quelques étudiants travaillent pour l'ensemble du groupe. Le travail personnel inclus en séance en présence des enseignants-tuteurs est une solution efficace pour favoriser le travail de tous les étudiants en amont du travail en groupe ;

- L'organisation des séquences APP est faite sous la forme de deux défis, l'un du type « découverte », l'autre du type « calculatoire ». Devant la difficulté des concepts (liés aux outils mathématiques notamment) qui devaient être acquis par les étudiants, nous avions prévu une organisation en trois temps : séance Défi $n^{\circ} 1$ « découverte » / Séance plénière (cours sur les outils mathématiques) / séance Défi $n^{\circ} 2$ «calculatoire ». Le positionnement intermédiaire de ce cours devait permettre une prise en main rapide des outils mathématiques. Le $1^{\mathrm{er}}$ défi dit de « découverte » permet de prendre conscience du nouveau phénomène et de le décrire simplement «avec les mains ». Ce $1^{\text {er }}$ défi correspond aux premiers niveaux d'apprentissage de la pyramide de Bloom (Bloom et Krathwohl, 1957), correspondant à la connaissance et la compréhension. Le $2^{\mathrm{e}}$ défi dit « calculatoire » permet d'aller plus en profondeur en menant des calculs en s'appuyant sur les méthodes de résolution de l'équation de Schrödinger qui régit la mécanique quantique. Cette $2^{\mathrm{e}}$ étape vise des types d'apprentissage plus élevés dans la taxonomie de Bloom. Ici, le $2^{\mathrm{e}}$ défi vise l'application de la théorie dans de nouveaux systèmes et l'analyse des résultats obtenus. Cette approche sous la forme de deux défis évite une compréhension superficielle des phénomènes. De plus, elle permet d'acquérir les méthodes de résolution indispensables pour une analyse poussée des phénomènes et dans certaines situations, cela permet d'être même prédictif. Le dispositif ne prévoit pas d'aller plus loin dans l'apprentissage au sens de Bloom. Cependant, il est possible $\mathrm{d}^{\prime}$ imaginer ajouter un $3^{\mathrm{e}}$ défi à chacune des quatre séquences APP qui pourrait ainsi viser les deux derniers étages de la pyramide Bloom, correspondant à la synthèse et à l'évaluation ; 
- Dans cette première édition, les comptes rendus de chaque défi étaient rédigés sur papier par un «secrétaire » désigné par l'ensemble du groupe et tournant à chaque séance. Ainsi sur l'ensemble du dispositif, chaque étudiant avait été responsable de la rédaction d'une solution. Cette solution était corrigée à la fin de chaque séance et rendue «au groupe ».

- Une évaluation formative à titre individuel est faite sous la forme d'un QCM à la fin de chaque séquence APP. Cette auto-évaluation faite en amont a pour objectif de préparer l'étudiant à l'examen individuel appelé «Quick-test » qui a lieu à la fin de chacune des 4 séquences APP. L'ensemble des notes obtenues aux «Quick-test» comptent pour $20 \%$ de la note finale. Les exercices sont également proposés dans le livret à titre d'entraînement avec les solutions, mais il n'y avait pas de correction en classe lors de la $1^{\text {re }}$ édition.

\subsection{Description des évolutions du dispositif}

Pour la seconde et la troisième expérimentation, nous avons mis en place des changements basés sur le ressenti des étudiants suite à l'évaluation (décrite en 3.3.) réalisée au terme de la première et de la seconde année.

a) Évolution en $2^{\mathrm{e}}$ année d'expérimentation

Lors de la $2^{\mathrm{e}}$ année d'expérimentation, le compte rendu écrit du groupe a évolué sous la forme d'un tableau qui se devait d'être bien organisé. Ce tableau est photographié par les étudiants et ensuite mis en ligne sur une plateforme partagée. Ce faisant, il peut être vu des autres groupes et être corrigé/évalué par les tuteurs.

La séance plénière était placée entre le défi $n^{\circ} 1$ et le défi $n^{\circ} 2$ lors de la première année d'expérimentation. Pour la $2^{\mathrm{e}}$ année, il a été décidé de déplacer cette séance à la fin de la séquence APP pour devenir une séance de clôture, comme cela est décrit dans la Figure 2. Le contenu de cette séance de clôture a été redéfini en deux temps distincts pour ancrer les nouvelles connaissances : un temps de synthèse des principaux points à acquérir et un temps d'exercisation. Les exercices préalablement faits à la maison sont travaillés en groupe et un exercice d'examen est corrigé pendant cette séance afin de préparer les étudiants à l'examen final. 


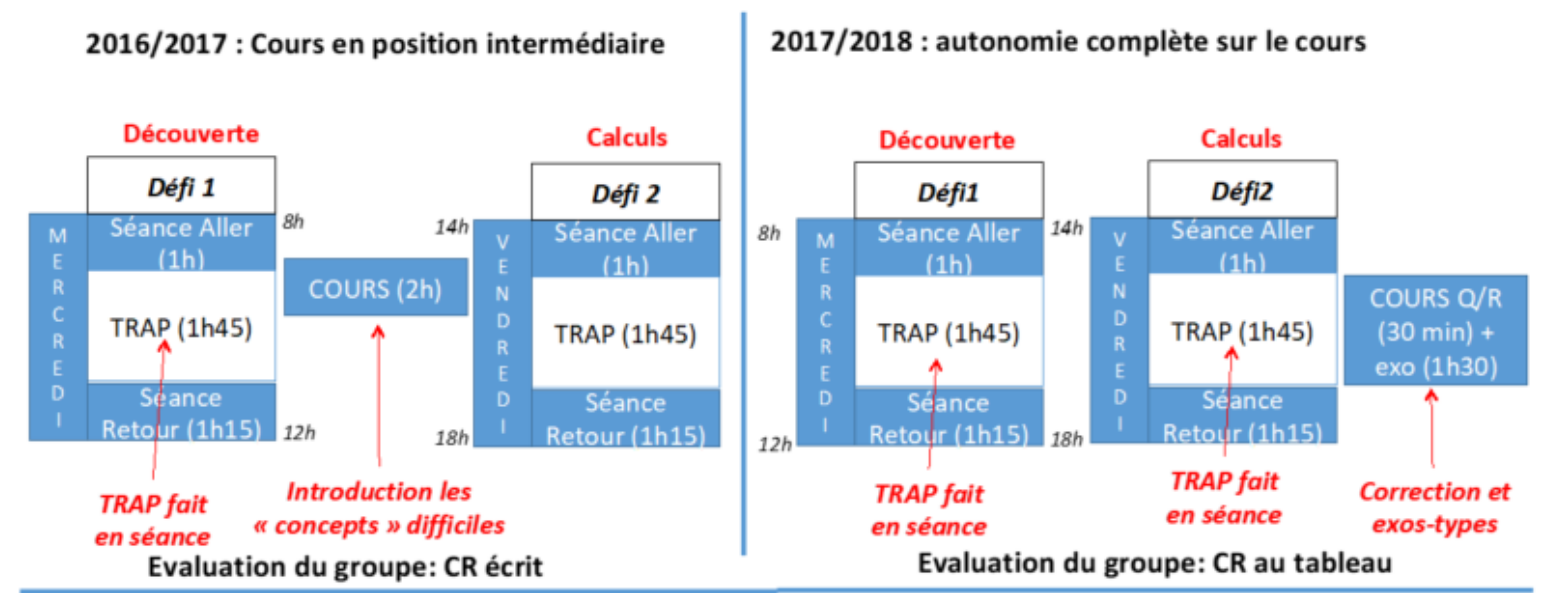

Figure 2 : Évolution de l'organisation d'une séquence APP dans le cadre du dispositif mis en place à Polytech Grenoble « De la molécule aux matériaux solides » : la séance plénière et le type de compte rendu (CR) ont été modifiés pour améliorer le dispositif

b) Évolution en $3^{\mathrm{e}}$ année d'expérimentation

D'autres améliorations ont été mises en œuvre lors de la $3^{\mathrm{e}}$ année d'expérimentation (20182019). Principalement, la formulation de l'ensemble des situations-problèmes ainsi que l'organisation et le contenu des ressources ont été retravaillés, afin de mieux cibler les apprentissages et de permettre aux étudiants de travailler avec beaucoup plus d'autonomie.

\subsection{Description de l'évaluation}

Chaque année, les étudiants ont réalisé une évaluation à l'aide d'un questionnaire en ligne. Elle était positionnée entre la fin des séances APP et l'examen terminal en assurant l'anonymat des réponses. Le questionnaire comprend une question d'appréciation globale, 22 autres questions sur 4 grands thèmes (les aspects généraux, le travail de groupe, le tutorat et les processus d'évaluation) et des espaces d'expression pour des commentaires libres (points positifs, points négatifs et suggestions) (voir la Figure 3). 


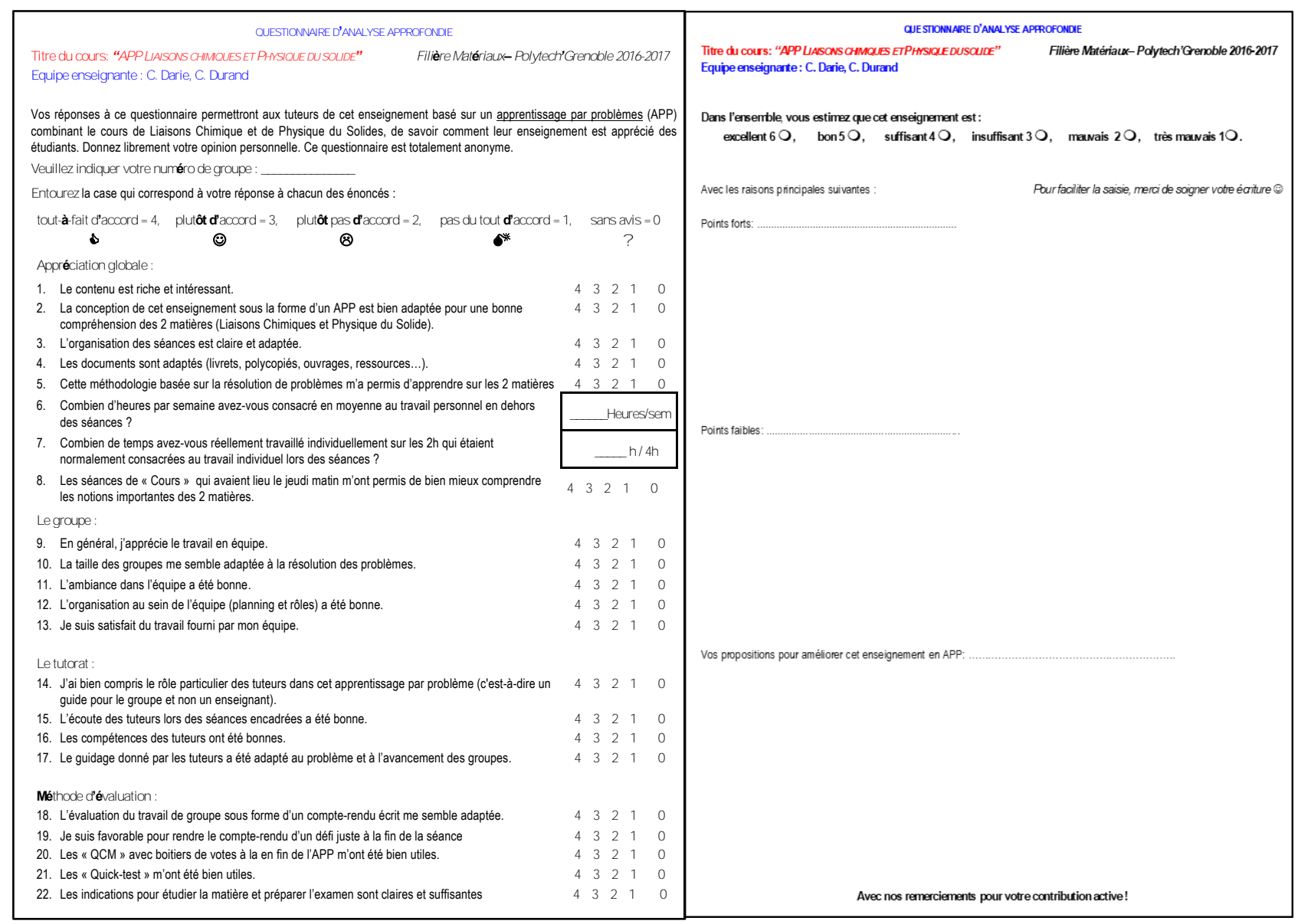

Figure 3 : Questionnaire d'évaluation du dispositif APP mis en place

La Figure 3 montre l'ensemble du questionnaire et la formulation de chacune des propositions. Nous avons fait le choix de proposer des affirmations positives pour chaque item à évaluer. Les étudiants avaient le choix de répondre par 4 réponses possibles : « tout à fait d'accord », " plutôt d'accord », «plutôt pas d'accord » et " pas du tout d'accord». Le choix médian n'était pas proposé, ce qui oblige les étudiants à se positionner soit « pour », soit « contre » la proposition. Néanmoins, on propose aussi le choix «sans avis » pour permettre aux étudiants de ne pas répondre s'ils n'ont pas un avis sur la proposition donnée (Ricci, 2009). Pour la question sur l'appréciation globale, nous avons proposé une échelle numérotée de 1 («Très mauvais ») à 6 («Excellent ») afin de mesurer plus finement le ressenti des étudiants en évitant ici aussi la possibilité de choisir une réponse médiane pour obliger les étudiants à se positionner. 


\section{Analyses : retours des étudiants sur le dispositif et bilan de la réussite aux examens}

\subsection{Retours des étudiants}

Ce nouveau dispositif pédagogique a été évalué par les étudiants pendant les trois années en utilisant le même questionnaire décrit ci-dessus et correspondant à la Figure 3. Un taux de réponse entre $83 \%$ et $98 \%$ a pu être obtenu pour un effectif d'environ 50 étudiants en aménageant dans le planning un temps dédié de 30 minutes pour cette évaluation. Ce taux de réponse élevé permet de considérer les réponses suffisamment fiables pour être exploitées.

La Figure 4 montre le résultat de la question sur l'appréciation générale. Une majorité d'étudiants adhère au nouveau dispositif dès la première année d'expérimentation puisque $70 \%$ jugent positivement cet enseignement. Une amélioration continue est obtenue à l'issue de la $2^{\mathrm{e}}$ et $3^{\mathrm{e}}$ année d'expérimentation du dispositif APP avec une moyenne qui augmente de 4,6 à 4,9/6, puis à 5,1/6. Également, les étudiants considérant cet enseignement comme « excellent ou bon » augmente aussi progressivement : $70 \%, 80 \%$, puis $88 \%$ avec une progression significative des étudiants ayant répondu «excellent» qui s'accroît de 5 à $30 \%$. La part d'étudiants réfractaires ayant répondu « insuffisant » ou « mauvais » se réduit aussi passant de $13 \%$ à $2 \%$. À l'issue de la $3^{\mathrm{e}}$ année, nous pouvons considérer que la quasi-totalité des étudiants adhèrent au dispositif démontrant que les changements apportés ont été bénéfiques.

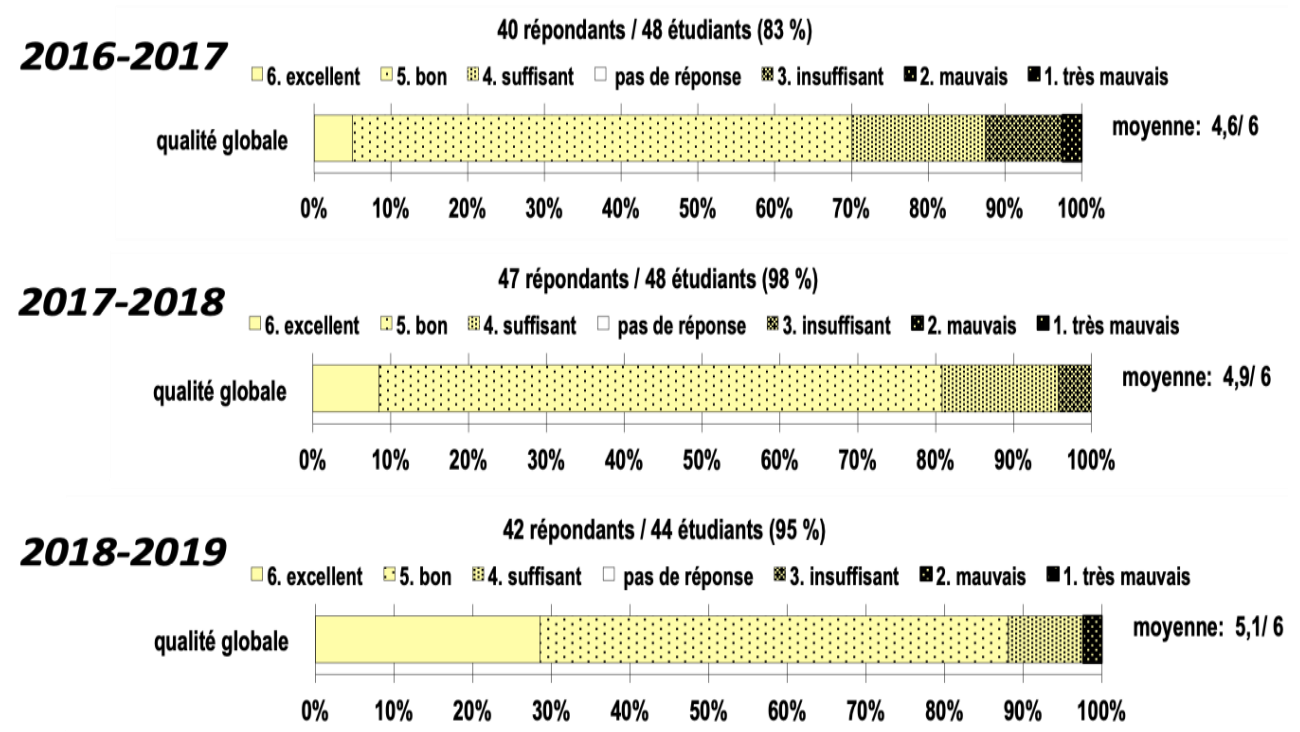

Figure 4 : Évaluation globale de cet enseignement en APP faite auprès des étudiants sur trois années consécutives

Pour mieux comprendre cette évolution, nous allons nous intéresser aux résultats des 22 questions du questionnaire qui sont représentées sur la Figure 5. Le taux de réponse en bleu 
foncé et bleu clair montre respectivement le taux d'étudiants « très satisfaits » et « satisfaits », alors que le rouge clair et rouge foncé sont relatifs respectivement aux étudiants «plutôt pas satisfaits » et « pas du tout satisfaits ».

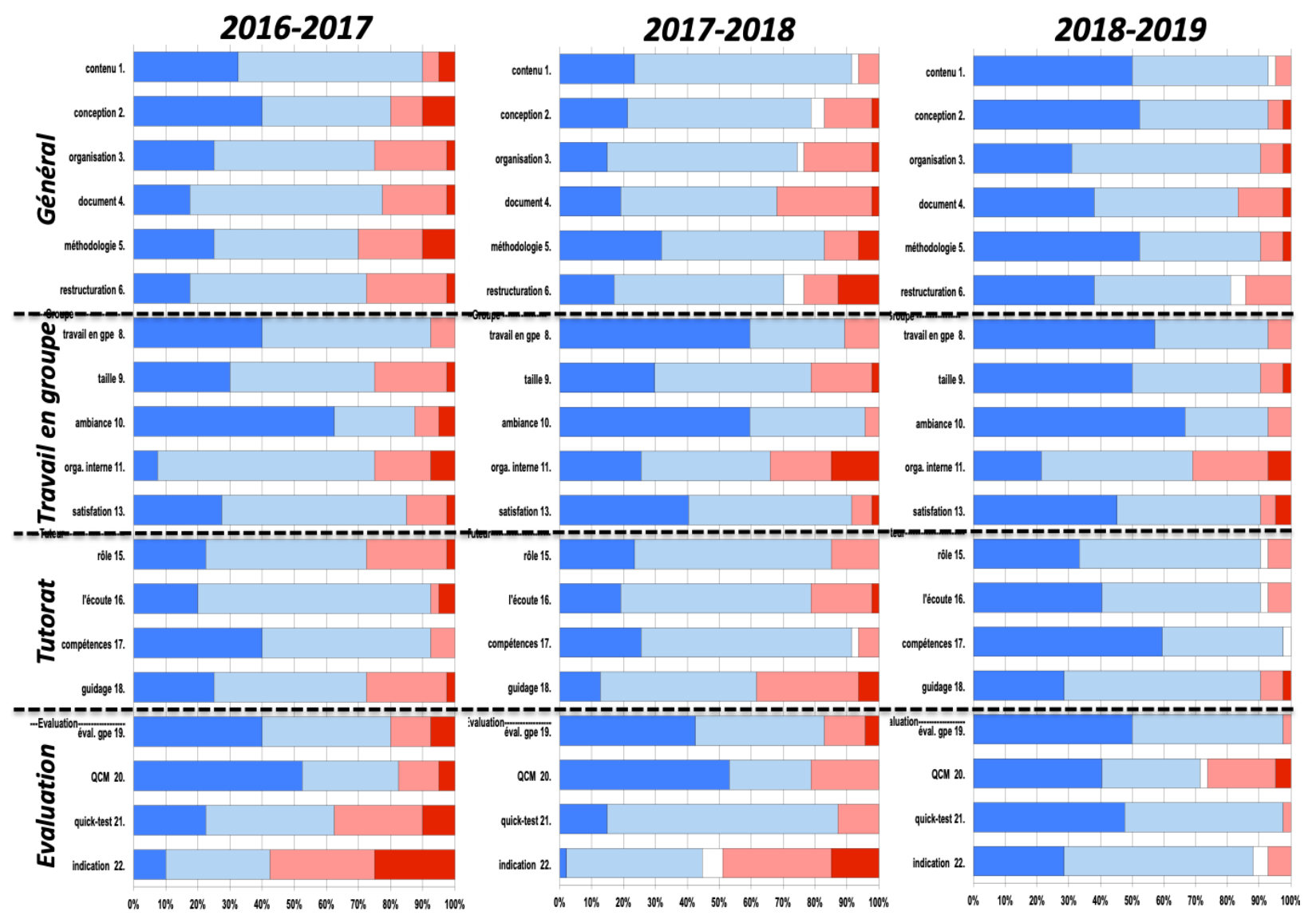

Figure 5 : Évaluation détaillée de cet enseignement en APP comprenant 22 questions autour de 4 grands

thèmes faite auprès des étudiants sur trois années consécutives (code couleur : bleu foncé : « très satisfait », bleu « satisfait », blanc « sans opinion », rouge « pas satisfait », rouge foncé « pas du tout satisfait »)

Sur les questions générales concernant le contenu, la conception, l’organisation, les documents, la méthodologie et la séance plénière (Q1-Q6), il y a une forte adhésion des étudiants avec un taux de satisfaction supérieur à $70 \%$ sur les trois années d'expérimentation. Nous observons une réelle progression pour la $3^{\mathrm{e}}$ année avec au moins $30 \%$ de réponses du type «Très satisfait ». En particulier, il y a eu un gain important sur l'adhésion à la méthodologie (Q5) et sur la séance plénière de restructuration (Q6). Nous attribuons cette amélioration à la nouvelle organisation où la séance plénière a été déplacée à la fin de la séquence APP (voir la Figure 2). Avec l'organisation initiale où la séance plénière était placée entre les deux défis, nous observions que les étudiants étaient en majorité passifs et peu intéressés, car ils retrouvaient les 
« habitudes » du cours classique. Cela était assez frustrant en comparaison de ce qui était vécu pendant les séances du type «Défi ». Ainsi, le cours a finalement été intégré aux ressources disponibles et a été réécrit pour pouvoir être travaillé en autonomie pendant le travail personnel. Avec la séance plénière placée à la fin, l'enseignant fait un bilan du travail des groupes et une synthèse des points importants du cours. Pendant cette séance, un travail en groupe est également proposé pour résoudre des exercices-types avec mise à disposition des corrections (un exercice est notamment extrait de l'ancien examen). Cette séance vient en soutien aux étudiants pour les apprentissages et pour préparer l'examen. Ce choix a été fait à la suite de l'évaluation faite à la première année d'expérimentation, où $20 \%$ des étudiants réclamaient dans les commentaires libres de faire des exercices d'entraînement afin d'être mieux préparés à l'examen. Bien que cette nouvelle organisation ait été proposée dès la $2^{\mathrm{e}}$ année, une amélioration de la perception de ce cours de restructuration (Q6) est visible seulement en $3^{\mathrm{e}}$ année. En effet, le contenu et l'organisation de cette séance ont été retravaillés en $3^{\mathrm{e}}$ année pour être pleinement exploités comme une séance de clôture de la séquence APP en faisant un bilan sur le cours et les exercices-types bien ciblés. Nous pensons que cette nouvelle organisation contribue aussi indirectement à l'amélioration de l'adhésion des étudiants à certains autres items comme la conception $(\mathrm{Q} 2)$ et l'organisation $(\mathrm{Q} 3)$.

Concernant les questions sur le travail de groupe (Q8 à Q13), les étudiants plébiscitent le travail en groupe. Il y a une vraie entraide mutuelle entre les étudiants sur cet enseignement, car le contenu théorique est difficile à comprendre tout seul. Il est intéressant de noter que le travail de groupe est mieux évalué à l'issue de la seconde et de la troisième année avec un taux d'étudiants se déclarant « très satisfaits » à $60 \%$ sur le travail de groupe (Q8), alors qu'il était de $40 \%$ la première année. Ceci est attribué en grande partie à la mise en place des comptes rendus sur tableau qui renforce le travail en équipe notamment lors de la construction du tableau. Ce point est important, car lorsque la solution était rédigée sur feuille, l'implication du groupe dans la rédaction était dans la plupart des cas nulle. En effet, l'étudiant avec le rôle de secrétaire rédigeait seul la solution «au propre » en dehors du temps de classe. Il semblait également que les solutions corrigées ne circulaient pas nécessairement au sein des groupes.

La rédaction de la solution sur le tableau mise en place dès la $2^{\mathrm{e}}$ année a l'avantage d'associer plus facilement l'ensemble du groupe à la rédaction (d'autant plus que les étudiants sont encouragés à tous contribuer à la construction du tableau) et de limiter dans le temps de classe la rédaction de la solution. C'est également un support qui permet au tuteur d'évaluer en direct le niveau de compréhension du groupe et lui donne l'occasion d'intervenir comme catalyseur 
dans la structuration et l'organisation de la pensée collective pour établir la solution. Ce support est aussi plus facile à lire et à évaluer, car le format du tableau impose d'être synthétique. C'est d'autant plus pratique que le nombre de groupe à tutorer et à évaluer est grand pour le tuteur.

L'organisation interne des groupes n'est pas toujours bien jugée avec 30-25\% d'étudiants insatisfaits (Q11) sans progression notable sur les 3 années. Ceci est lié au travail en groupe où certains étudiants s'investissent peu et pénalisent l'avancée du groupe. Les étudiants «peu investis » même en très faible nombre influent négativement sur l'organisation interne de certains groupes. Il faut néanmoins composer avec tous les profils d'étudiants et le tuteur peut jouer un rôle important pour favoriser l'implication des étudiants récalcitrants, sans avoir de garantie de succès. Les étudiants peu investis sont plus facilement détectables pendant la phase du travail personnel, où l'enseignant-tuteur peut intervenir en tête-à-tête pour résoudre un problème de compréhension où encourager l'étudiant à s'investir.

Le tutorat (Q15-Q18) est très apprécié avec un taux de satisfaction sur l'écoute et les compétences supérieur ou égal à $80 \%$. Par contre, il est intéressant de noter que le guidage par les tuteurs est beaucoup plus mal évalué avec 40-30\% des étudiants insatisfaits en $1^{\text {re }}$ et $2^{\mathrm{e}}$ année (Q18). Ce ressenti est lié en partie à la méthode APP où les enseignants-tuteurs reformulent les questions et guident les étudiants sans répondre directement aux questions posées. Pourtant, le guidage du tuteur a été bien mieux apprécié en $3^{\mathrm{e}}$ année pour atteindre $90 \%$ des étudiants satisfaits, car la réécriture du cours et des situations-problèmes a permis aux tuteurs d'être moins sollicités et d'avoir plus de « vrai » temps avec chacun des groupes.

Sur les questions autour de l'évaluation (Q19-Q22), nous notons que les QCM formatifs sont appréciés (plus de $50 \%$ d'étudiants «Très satisfaits »). L'évaluation du travail de groupe est aussi très bien jugée, mais ceci est lié en grande partie aux notes plutôt élevées attribuées par les tuteurs (moyenne de 16/20). Ces «bonnes » notes qui reflètent le travail fourni par les groupes ont aussi l'avantage de valoriser le travail de groupe (même si ce travail collectif compte pour seulement $5 \%$ de la note finale). Une forte progression du ressenti des étudiants a été obtenue sur les quick-tests (qui compte pour $20 \%$ de la note finale) et sur l'évaluation finale (qui compte pour $75 \%$ de la note finale) en $2^{\mathrm{e}}$ et $3^{\mathrm{e}}$ année d'expérimentation. Nous pensons que cette amélioration est liée à la séance de clôture qui fait le bilan des apprentissages et permet l'exercisation. Notamment, en ciblant mieux les exercices préparés en intégrant des exercices issus d'examens des années antérieures et en donnant systématiquement les corrections, les étudiants se sont sentis bien mieux préparés aux quick-tests et à l'examen 
terminal comme le montre les réponses à Q21 et Q22 avec respectivement, $95 \%$ et $90 \%$ des étudiants satisfaits.

Enfin, certains étudiants estiment dans les commentaires libres que le temps du travail personnel (TRAP) est trop long ( $1 \mathrm{~h} 45)$ et que les réponses obtenues de la part des tuteurs ne sont pas suffisantes. Néanmoins, chercher à répondre à ce type de reproches peut impacter négativement le dispositif APP. En effet, la réduction du travail personnel réduit la compréhension du cours et dégrade la qualité de travail préparatoire qui est pourtant une condition sine qua non pour obtenir un travail d'équipe conséquent et efficace.

Après trois années d'expérimentation, nous avons obtenu une très bonne adhésion des étudiants au nouveau dispositif pédagogique grâce à la nouvelle organisation et au travail de réécriture du cours et des situations-problèmes. Dans les commentaires libres, beaucoup d'étudiants soulignent le côté «ludique », le côté plus efficace que le cours magistral et la meilleure compréhension. Citons à titre d'exemples ces retours d'étudiants : "Bonne ambiance de travail qui donne envie de s'investir », " Travail en groupe plutôt qu'être passif en amphi et qui permet de bien comprendre les cours », "Je pense que plus de cours devraient être donnés sous cette forme, beaucoup plus pédagogique et qui permet de ressortir avec beaucoup plus de connaissances ». Des marges d'amélioration sont encore possibles, notamment sur l'organisation interne des groupes et sur le suivi ciblé des étudiants en difficulté (facilement détectables par les notes obtenues aux quick-tests) qui se démotivent au fur et à mesure des séquences APP.

\subsection{Apprentissage et réussite aux examens}

Un des objectifs du nouveau dispositif pédagogique APP visait à modifier la représentation du modèle de l'atome au sein d'une molécule ou d'un solide et que cette nouvelle vision soit durablement ancrée chez les étudiants. Il est difficile d'établir rigoureusement si ce changement de représentation s'est bien opéré. Pour cela, il conviendrait de mettre en place une étude spécifique sur les étudiants ayant et n'ayant pas suivi le dispositif APP pour «mesurer » ce changement. En tant qu'enseignants, nous avons l'impression que ce changement de représentation est présent, mais s'opère plutôt pour les étudiants qui sont en situation de réussite. On peut regretter que cela ne touche pas tous les étudiants, mais nous considérons que c'est déjà un point positif que ce changement de représentation puisse être effectif, car c'était un point difficile avec un enseignement traditionnel et cloisonné. 
Au-delà du changement de représentation de l'atome non isolé, nous pouvons comparer la réussite aux examens avant et après la mise en place du dispositif pédagogique. Pour pouvoir faire cette comparaison, nous avons récolté les notes des deux examens terminaux correspondant à celui de «Liaisons Chimiques » et celui de «Physique du Solide » pour la période 2014/2015 et 2015/2016 où l'enseignement a été fait de manière classique sous la forme de cours/TD, et pour la période 2016/2017, 2017/2018 et 2018/2019 où l'enseignement a été fait en APP en fusionnant ces deux matières. La difficulté des examens sur l'ensemble de la période 2014-2019 est comparable, car les exercices demandés sont très similaires et ont été rédigés et corrigés par les deux mêmes enseignants. Nous faisons l'hypothèse que le niveau des cohortes d'étudiants est similaire d'une année sur l'autre, car les origines des étudiants et les modes de sélection sont identiques. Avec un effectif de 46 étudiants en moyenne sur deux cours différents pendant une période de 5 ans, nous avons pu récolter plus de 460 notes permettant d'avoir une statistique conséquente. La Figure 6 montre le pourcentage d'étudiant ayant obtenu une note (comprise entre 0 et 20) obtenue à l'examen avant (période 2014-2016) et après (période 2016-2019) la mise en place des APP.

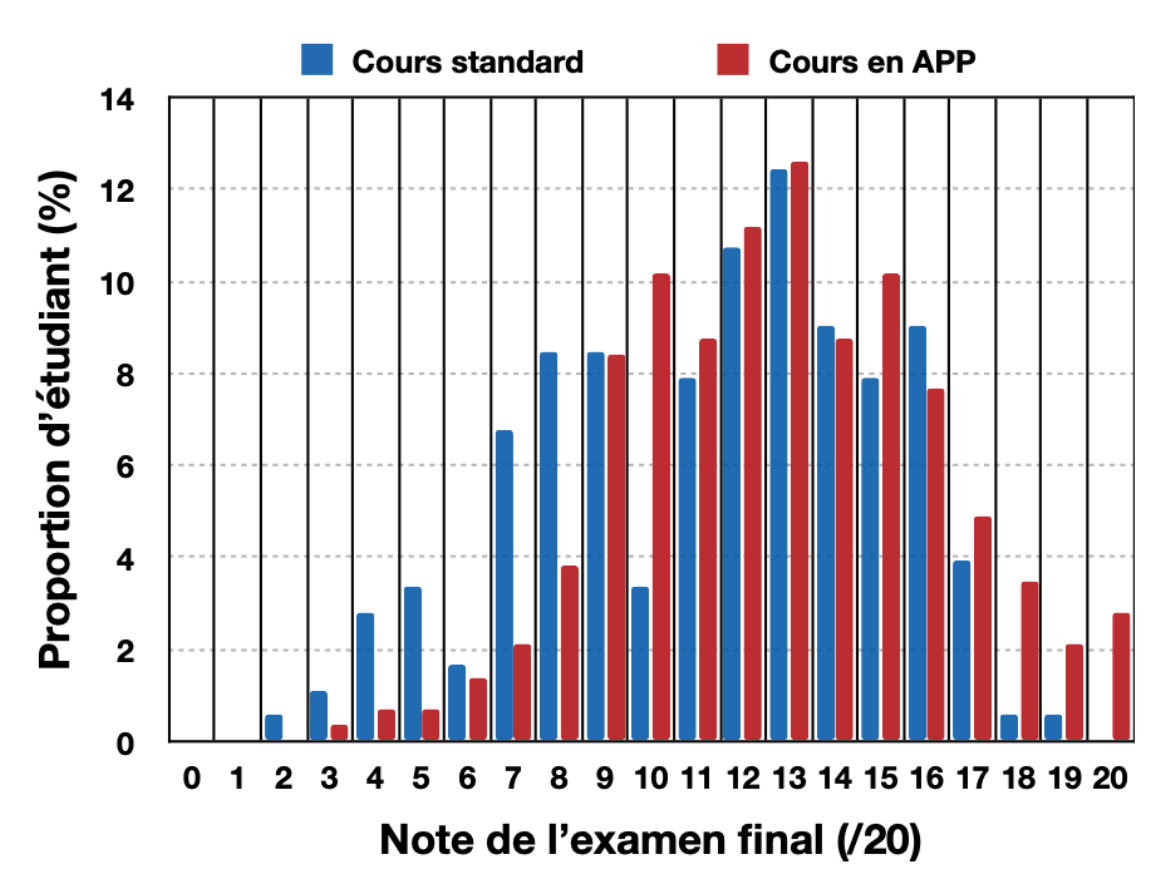

Figure 6 : Répartition en pourcentage des étudiants de la note obtenue à l'examen terminal pour les deux cours «Liaisons chimiques » et «Physique du Solide» sur une période de 5 ans. L'histogramme bleu correspond aux notes obtenues sur la période 2014-2016 après un enseignement classique (Cours/TD), alors que l'histogramme rouge correspond aux notes obtenues sur la période 2016-2019 après la mise en place du dispositif APP 
Sur la Figure 6, nous constatons une amélioration notable des résultats après la mise en place des APP qui se perçoit aussi à partir de l'évolution de la moyenne des examens terminaux qui passe de 11,36/20 à 12,29/20 (+0,93 point) après la mise en place des APP. La comparaison de la répartition des notes sur la Figure 6 est instructive. D'excellentes notes $(\geq 18 / 20)$ sont obtenues par 8,5\% des étudiants, alors que ces notes étaient quasiment inaccessibles avec un enseignement traditionnel $(1,1 \%)$. Par ailleurs, beaucoup moins d'étudiants obtiennent de très mauvaises notes $(\leq 7 / 20)$ quand l'enseignement est fait avec le dispositif APP. La Figure 6 montre très clairement que la réussite à l'examen n'a pas régressé avec le passage au nouveau dispositif pédagogique, mais au contraire le dispositif a permis de limiter le nombre d'étudiants « décrocheurs » ayant une note inférieure ou égale à 7/20. Avant l'APP, $15 \%$ des étudiants sont « décrocheurs », alors que ce taux se réduit à $5 \%$ après la mise en place de l'APP. À noter que la plupart du temps, ces « décrocheurs » n'ont pas bien respecté les consignes du dispositif pédagogique, ce qui leur a causé préjudice pour leur apprentissage. Si on considère la répartition des étudiants qui ont moins que la moyenne $(<10 / 20)$, on comptabilise $1 / 3$ des étudiants $(34 \%)$ qui sont dans cette situation avant les APP, alors que cette proportion se réduit à $1 / 6$ des étudiants $(17 \%)$ après. À partir de cette analyse, la proportion d'étudiants en difficultés avec une note inférieure à 10/20 est divisée par deux avec le dispositif APP, ce qui constitue un point très positif.

Même si une amélioration des résultats n'est pas toujours aussi nettement observée dans la littérature, notre étude va dans le même sens que beaucoup d'autres travaux qui mesurent des effets bénéfiques en comparant un enseignement fait de manière traditionnelle et fait sous la forme d'un APP sur des cohortes d'étudiants comparables (Frenay, Galand et Bourgeois, 2007 ; Galand, Frenay et Raucent, 2012 ; Selçuk, Sezgin et Serap, 2013 ; Martin et Padula, 2018).

\section{Bilan et perspectives}

Cette coopération disciplinaire a permis un regard croisé sur la description de l'atome non isolé avec une vision de chimiste d'une part et de physicien d'autre part. Cette double vision a permis de mieux comprendre les difficultés que les étudiants pouvaient rencontrer et a amené à proposer une description continue des concepts sous la forme d'un nouveau dispositif pédagogique avec un séquençage sous forme d'APP. Après trois ans d'expérimentation, nous avons identifié des solutions pratiques qui ont permis d'améliorer concrètement le dispositif. Nous pouvons citer le compte rendu sur tableaux, l'intégration du travail personnel dans les 
séances, la mise en place d'une séance de clôture avec bilan des travaux de groupe, synthèse du cours et temps d'exercisation ciblé. Ces choix pédagogiques ont été appréciés des étudiants avec plus de $85 \%$ d'entre eux qui évaluent positivement le nouveau dispositif pédagogique. Une amélioration des résultats à l'examen terminal a aussi été obtenue avec une augmentation de la moyenne $(+0,9)$ et une diminution de moitié du nombre d'étudiants ayant une note inférieure à 10/20.

La suite envisagée à ce travail est de pousser encore plus loin l'expérimentation de la fusion en intégrant l'enseignement de «Physique des Semi-conducteurs » qui s'inscrit à la suite du cours de «Physique du Solide ». Cette voie a justement été mise en place dès 2019 en développant trois nouveaux APP qui s'intègrent dans la continuité des séquences APP précédentes tout en abordant le contenu du cours de physique des semi-conducteurs :

- APP 5 : «Vive le dopage »;

- APP 6 : «La jonction P-N, une idée lumineuse ! »;

- APP $7:$ : Le transistor MOS : une révolution infiniment petite».

Cette approche globale sous la forme de 7 APP permet une continuité : de la simple molécule jusqu'aux matériaux semi-conducteurs et leurs applications complexes. Il serait certainement intéressant de réaliser un examen commun sur ces trois matières pour conserver cette idée de la continuité des notions. Plus largement, cette expérience doit nous encourager à décloisonner les matières pour donner plus de sens à nos formations.

\section{Références bibliographiques}

Aguirre, E., Jacqmot, C., Milgrom, E., Raucent, B., Soucisse, A. et Vander Borght, C. (2000). Devenir ingénieur par apprentissage actif. Actes $d u 1^{\text {er }}$ colloq. pédagogie par proj. dans l'enseignement supérieur, 1-7.

Bachelard, G. (1938). La formation de l'esprit scientifique. Paris : Vrin.

Bédard, D. et Raucent, B. (2015). Les innovations pédagogiques en enseignement supérieur : pédagogies actives en présentiel et à distance. Revue internationale de pédagogie de l'enseignement supérieur, 31(1), 1-3.

Bédard, D., Lison, C., Dalle, D. et Boutin, N. (2010). Predictors of student's engagement and persistence in an innovative PBL curriculum: Applications for engineering education. Int. J. Eng. Educ., 26(3), 511-522.

Bédard, D., Bibeau, J., Pilon, C. et Turgeon, A. (2020). L'espace expérientiel : une pédagogie interactive. Les annales de QPES, 1(1), 1-26. 
Bloom, B. S. et Krathwohl, D. R. (1956). Taxonomy of Educational Objectives: The classification of educational goals, Comité d'examinateurs de collèges et d'universités, Longmans

Boilevin, J.-M. (2005). Enseigner la physique par situation problème ou par problème ouvert. Aster, 40, 13. https://doi.org/10.4267/2042/8854

Boutin, N., Dalle, D., Lachiver, G. et Mabilleau, P. (2008). L'apprentissage par problèmes et par projets pour le développement des compétences professionnelles de l'ingénieur: l'exemple des programmes de génie électrique et de génie informatique de l'Université de Sherbrooke. J3eA, 7, 1029. https://doi.org/10.1051/j3ea:2008028

Celik, P., Onder, F. et Silay, I. (2011). The effects of problem-based learning on the students' success in physics course. Procedia - Soc. Behav. Sci., 28, 656-660. https://doi.org/10.1016/j.sbspro.2011.11.124

Frenay, M., Galand, B. et Bourgeois, É. (2007). L'approche par problèmes et par projets : effets de dispositifs pédagogiques actifs dans l'enseignement universitaire. Un enseignement démocratique de masse - Une réalité qui reste à inventer, 287-310.

Galand, B., Bourgeois, É. et Frenay, M. (2005). The impact of a PBL curriculum on students' motivation and self-regulation. Les Cah. Rech. du GIRSEF, 37, 1-16.

Galand, B., Frenay, M. et Raucent, B. (2012). Effectiveness of problem-based learning in engineering education: A comparative study on three levels of knowledge structure. Int. J. Eng. Educ., 28(4), 939-947.

Kittel, C. (2007). Physique de l'état solide. Paris : Édition Dunod.

Martin, P. et Padula, P. (2018). Innovation pédagogique à l'université : comparaison entre apprentissage par problèmes et cours traditionnel. Rev. Int. pédagogie l'enseignement supérieur, 34(3). https://doi.org/10.4000/ripes.1574

McQuarrie, D. A. et Simon, J. D. (2000). Chimie physique - approche moléculaire. Paris : Éd. Dunod.

Mundilarto, H. I. (2017). Effect of problem-based learning on improvement physics achievement and critical thinking of senior high school student. J. Balt. Sci. Educ., 16(5), 761-779.

Raucent, B., Braibant, J., De Theux, M.- N., Jacqmot, C., Milgrom, É., Vander Borght, C. et Wouters, P. (2004). Devenir ingénieur par apprentissage actif: compte rendu d'innovation. Didaskalia, 24, 1-19.

Raucent, B. et Milgrom, É. (2013). Guide pratique pour une pédagogie active: les apprentissages par problèmes et par projets. INSA Toulouse et École Polytechnique de Louvain. ISBN : 978-2-87649-059-8

Ricci, J. (2009). Chapitre 2. La qualité de l'enseignement au supérieur : un vaste chantier qui déborde largement de l'opinion des étudiants. Dans M. Romainville (dir.), L'évaluation de l'enseignement par les étudiants : Approches critiques et pratiques innovantes (p. 3555). Louvain-la-Neuve : De Boeck Supérieur. 
Selçuk, G., Sezgin, C. et Serap, S. M. (2013). A comparison of achievement in problem-based, strategic and traditional learning classes in physics. Int. J. New Trends Educ. their Implic., 4(1), 154-164.

Svinicki, M. et McKeachie, W. J. (2014). McKeachie's teaching tips (14 éd.). Belmont, CA: Wadsworth. 